\title{
Giant nonlinear response of superconducting single crystal niobium in a sweeping magnetic field.
}

\author{
M.I. Tsindlekht ${ }^{\mathrm{a} *}$, I. Felner ${ }^{\mathrm{a}}$, G. Jung ${ }^{\mathrm{b}} \ddagger$ \\ aThe Racah Institute of Physics, The Hebrew University of Jerusalem, \\ Jerusalem, 91904, Israel \\ ${ }^{\mathrm{b}}$ Department of Physics, Ben Gurion University of the Negev, \\ P.O.Box 653, 84105 Beer-Sheva, Israel
}

\begin{abstract}
Giant enhancement of the nonlinear response of a single crystal of $\mathrm{Nb}$ placed in a sweeping magnetic field has been experimentally observed. The rectified signal from $\mathrm{Nb}(T c=9.15 \mathrm{~K})$ has been measured by means of an inductive method as a function of temperature, dc field, dc field sweep rate, and the amplitude of ac field. The $\mathrm{Nb}$ sample was excited by an amplitude modulated ac field. Under a stationary regime, the rectified signal appears only for magnetic fields $\left(H_{0}\right)$ in the range $H_{c 2}<H_{0}<H_{c 3}$. However, when the dc field was swept slowly, the rectified signal appears at $H_{0}>H_{c 1}$. This experiment shows that the amplitude of the rectified signal is two orders of magnitude larger than the amplitude of the signal seen under stationary field conditions. Moreover, the amplitude of the rectified signal is a power function of the sweep rate, with the power exponent close to 1.
\end{abstract}

\section{Introduction}

Non-stationary phenomena in type-II superconductors have attracted attention since the middle sixties. Varying the dc magnetic field results in an increase of the ac losses in the mixed state 11]. Moreover, while sweeping a dc magnetic field between $H_{c 1}$ and $H_{c 2}$, a second harmonic signal appears in the response to the ac field excitation [2]. H. J. Fink [3] showed that the second harmonic signal reaches maximum at a sweep rate $d H_{0} / d t \approx \omega h_{0}$, where $\omega$ and $h_{0}$ are the frequency and amplitude of ac field respectively, and disappears when $d H_{0} / d t<<\omega h_{0}$.

Using high quality single crystal niobium, we have shown recently that nonlinear response to small external ac excitation field under stationary conditions, appears only in the surface superconducting state [4. In this paper we show that in non-stationary conditions even non-linear effects appear also for $H_{c 1}<H_{0}<H_{c 2}$. Moreover,

\footnotetext{
*The work at the Hebrew University was supported by 1998 Binational US-Israel Science Foundation grant and by the Klatchky foundation.

$\dagger$ e-mail address: mtsindl@vms.huji.ac.il

‡also with Instytut Fizyki PAN, Warszawa, Poland
}

the magnitude of this nonlinear non-stationary response is orders of magnitude higher than the response observed under stationary conditions [4] and, therefore, it will be referred to as "a giant response".

\section{Experimental}

A rectangular sample, $10 \times 3 \times 1 \mathrm{~mm}^{3}$, was cut out high purity single crystalline $\mathrm{Nb}\left(T_{c}=\right.$ $9.15 \mathrm{~K}$ and resistance ratio $\left.R_{300 K} / R_{10 K} \approx 300\right)$. The results of magnetization measurements of our sample were published elsewhere [A].

The sample was exposed to $\mathrm{dc} H_{0}$ and ac $h(t)$ magnetic fields applied parallel to the [100] crystalline direction which coincides with the longest dimension of the rectangular sample. The amplitude modulated ac field $h(t)=h_{0}(1+$ $\alpha \cos \Omega t) \cos \omega t$, where: $0<h_{0}<0.6 \mathrm{Oe}, \alpha \approx 0.9$, $\omega / 2 \pi=3.2 \mathrm{MHz}$, and $\Omega / 2 \pi=733 \mathrm{~Hz}$, was obtained by feeding the small primary copper coil from a high frequency generator operating in a constant current regime. The detailed description of the experimental setup was given elsewhere [4]. Sweeping the dc magnetic field was 
achieved by a normal metal solenoid of a commercial SQUID magnetometer system. The maximum available amplitude of the field sweep was 120 Oe and sweep rate $d H_{0} / d t$ up to $24 \mathrm{Oe} / \mathrm{s}$. After reaching the maximum field at a given sweep rate, the field was decreased at the same sweep rate. The nonlinear response has been measured inductively by means of a secondary pick-up copper coil wound on the primary coil.

Nonlinearity of a superconducting specimen immersed in an amplitude modulated ac field results in oscillations of the magnetic moment at the frequencies of the harmonics of the fundamental frequency $\omega$, and at the frequencies $\omega \pm \Omega$, as well as at the modulation frequency $\Omega$, and its harmonics. The pick-up coil transforms the oscillations of the magnetic moment into ac voltage signals. The signal was processed by a lock-in amplifier as a function of the experimental parameters such as: temperature, $H_{0}, \mathrm{dc}, d H_{0} / d t$, and ac field amplitude $h_{0}$.

The nonlinear response to a dc magnetic field sweep, i.e. the amplitude of the rectified signal $A_{\Omega}$ at the frequency $\Omega$, has been measured as follows. The sample was first cooled down to the required temperature at zero magnetic field. Next, $H_{0}$ was raised to $H_{s t}$, the actual sweep of $H_{0}$ was ramped-up, and eventually $A_{\Omega}$ was measured as a function of time. In the following we shall discuss only the phenomena associated with the increasing magnetic field sweep. The hysteretic behavior observed when the field was swept down will be discussed elsewhere.

\section{Results and discussion}

Figure 1 shows the field dependence of the rectified signal $A_{\Omega}$ measured at $\mathrm{T}=8.5 \mathrm{~K}$ for various $H_{s t}$ values. Both sweep rate and ac amplitude $h_{0}$ are constant. It is readily observed that for $H_{0}<H_{c 1}$ the response is negligible. However, for $H_{0}>H_{c 1}$ a slight increase in the signal appears. For $H_{0}$ close to $H_{c 2}$ the magnitude of the response grows significantly, and exceeds the stationary response measured in the same setup with the same sample by two orders of magnitude [4]. This result contradicts previous experimental observation [1,2] and theoretical predic- tions [3]. This is due to the fact that in our experiments $d H_{0} / d t<<\omega h_{0}$ and therefore, in accordance with Fink [3], $A_{\Omega}$ should be zero.

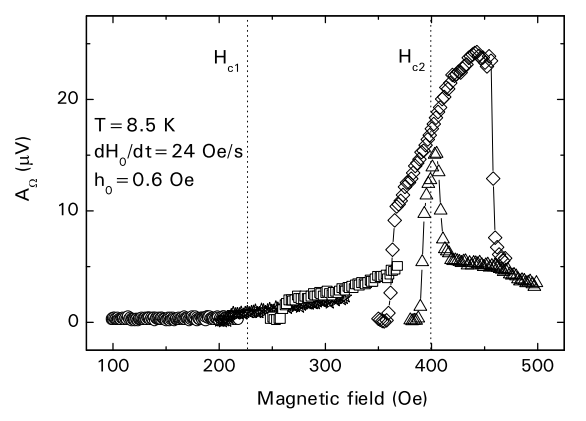

Figure 1. Rectified signal $A_{\Omega}$ vs. sweeping magnetic field at $8.5 \mathrm{~K}$. Different symbols correspond to different starting fields $H_{s t}$. Dotted lines indicate positions of $H_{c 1}=225$ Oe and $H_{c 2}=400$ Oe.

Further increase of $H_{s t}$ towards $H_{c 2}$ leads to a new phenomenon. Rapidly increasing $A_{\Omega}$ with a the maximum at $H_{0}>H_{c 2}$ sharply drops at $H_{0}=$ $H_{k}$. For $H_{0}>H_{k} A_{\Omega}$ remains almost constant for any $H_{s t}$ and then the signal gradually decreases to zero with $H_{0}$ approaching $H_{c 3} \approx 600 \mathrm{Oe}$. At the same time all hysteretic phenomena disappear when $H_{s t} \geq H_{c 2}$.

The value of $H_{k}$ at which $A_{\Omega}$ abruptly drops turned out to be strongly dependent on the experimental parameters such as $H_{s t}, h_{0}$ and $d H_{0} / d t$. We have found that with decreasing the sweep rate and/or with increasing the excitation amplitude and starting field, the characteristic field $H_{k}$ asymptotically approaches $H_{c 2}$. For example, Fig. 2 shows the dependence of $H_{k}$ on sweep rate $d H_{0} / d t$. The solid line is a best fit to $H_{k} \propto\left(d H_{0} / d t\right)^{2}$. We would like to emphasize that Fig. 2 presents some type of dynamical phase diagram. Namely it is clear that the solid line is a dynamic boundary between surface and bulk superconductivity.

The experiments revealed that the amplitude of the rectified signal increases with $d H_{0} / d t$. This 


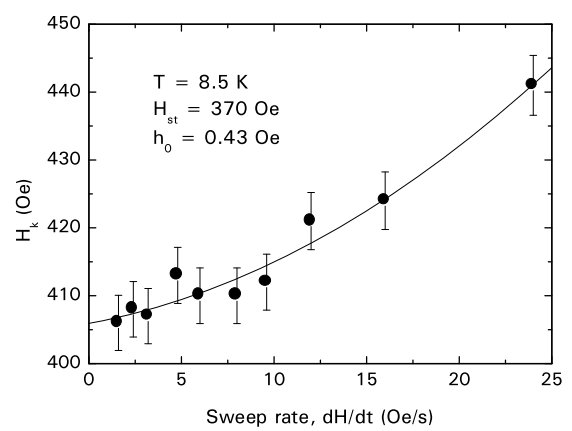

Figure 2. Characteristic field $H_{k}$ at which the rectified signal abruptly drops as a function of the sweep rate of the magnetic field.

dependence, determined for three different values of the $H_{0}$, is presented in Fig. 3. The power law fit to the data shows that $A_{\Omega} \propto\left(d H_{0} / d t\right)^{p}$ with the exponent $p=0.95 \pm 0.02$. The exponent $p$ does not depend on temperature, $h_{0}$ and $H_{s t}$. However as $H_{0}$ approaches the field at which $A_{\Omega}$ goes through a maximum, the exponent $p$ goes down, as illustrated in Fig. 3 for $H_{0}=415$ Oe, very close to the response maximum, Fig. 1.

The discrepancy between the observed phenomena and previously reported results can be understood considering a sand pile analogue of a critical vortex state [5]. Slow increase of the dc field leads to an avalanche like vortex array redistribution. All previous experiments were performed at low frequencies at which any modifications of the nonlinear response due to vortex avalanches cannot be observed since at low frequencies $d B / d t \gg \omega h_{0}$. At high frequencies vortex avalanches become effective. Namely at high frequencies $d B / d t \approx \omega h_{0}$ is possible. Moreover, vortex avalanches occur in the entire volume of the sample and not only in the surface layer. The increase of the active volume may result in a significant enhancement of the nonlinear response. The proposed model may qualitatively explain the appearance near $H_{c 2}$ of a sharp drop of the rectified signal at $H_{k}$. It is clear, that only a quantative theory will be able explain features observed experimentally in the present work. How-

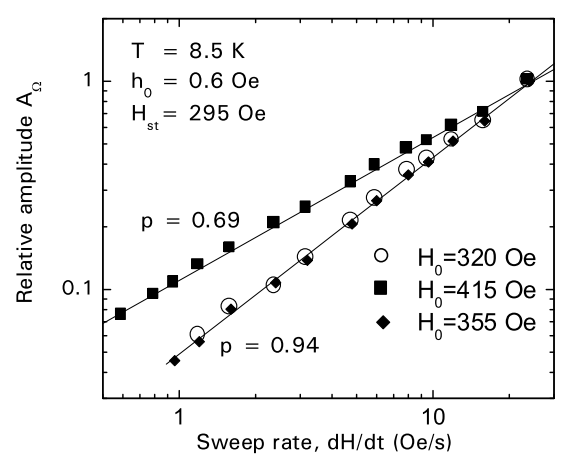

Figure 3. Amplitude of the rectified signal normalized to a maximum amplitude at a given $H_{0}$ as function of magnetic field sweep rate determined at three different values of the magnetic field $H_{0}$, see the legend.

ever, to the best our knowledge this theory does not exist as yet.

\section{REFERENCES}

1. E. Maxwell and W.P. Robbins, Phys. Lett. 19 (1966) 629.

2. S.A. Campbell, J.B. Ketterson, and G.B. Grabtree, Rev. Sci. Inst., 54 (1983) 1191.

3. H.J. Fink, Phys. Rev. 161 (1967) 417.

4. M.I. Tsindlekht, I. Felner, M. Gitterman, B.Ya. Shapiro, Phys. Rev. B, 62 (2000) 4073.

5. P.G. de Gennes, Superconductivity of Metals and Alloys ( W.A.Benjamin, New-York, 1966). 